\title{
Avanços e desafios da formação e qualificação dos profissionais e gestores do Sistema Único de Saúde em alimentação e nutrição
}

\author{
Progress and challenges in the training and \\ qualification of professionals and public \\ administrators of the Brazilian Unified National \\ Health System in food and nutrition
}

\author{
Avances y desafíos en la formación y calificación \\ de los profesionales y gestores del Sistema Único \\ de Salud brasileño en alimentación y nutrición
}

Aos desafios requeridos para qualificar as ações de alimentação e nutrição no Sistema Único de Saúde (SUS), abordados no artigo que trata dos 20 anos da Política Nacional de Alimentação e Nutrição (PNAN), insere-se o da formação dos profissionais/gestores, com importância estratégica. Já na 1ạ versão da PNAN, uma diretriz de capacitação foi explicitada como transversal a todas as demais, buscando “...preparar os recursos humanos para a operacionalização de um elenco básico de atividades, na perspectiva de promoção dos direitos humanos...” 1 (p. 27).

Na versão atual da PNAN (2012), a diretriz define como imprescindível "...a qualificação dos profissionais em consonância com as necessidades de saúde, alimentação e nutrição da população, sendo estratégico considerar o processo de trabalho em saúde como eixo estruturante para a organização da formação da força de trabalho" 2 (p. 45).

E a reconhece como "...uma necessidade histórica e estratégica para o enfrentamento dos agravos e problemas decorrentes do atual quadro alimentar e nutricional brasileiro” 2 (p. 46).

Desde a publicação da 1a versão da PNAN, portanto, vêm sendo desenvolvidas estratégias de formação em alimentação e nutrição no SUS. Essas estratégias foram - e são - revistas em observação da conjuntura epidemiológica, institucional, técnica e política, e do próprio crescimento da rede de atenção à saúde, em números e complexidade.

Houve, entre 1999-2006, um processo intenso de capacitação com ênfase nos atores estaduais equipes das coordenações/áreas técnicas das Secretarias Estaduais de Saúde (SES) responsáveis pela gestão das ações de alimentação e nutrição - para apoiar a implementação de ações, programas e sistemas de informação (Sistema Bolsa Alimentação, Bolsa Família, Sistema de Vigilância Alimentar e Nutricional, prevenção da desnutrição e de deficiências de micronutrientes, e outros), apostando na multiplicação destas capacitações nos municípios. Tais capacitações foram desenvolvidas diretamente pela equipe técnica da (então) Coordenação-Geral da Política de Alimentação e Nutrição (CGPAN), com deslocamento para estados e municípios ou por meio de eventos. Um exemplo: as Oficinas Macrorregionais de Capacitação em Alimentação e Nutrição para profissionais da atenção básica em saúde, realizadas entre 2001-2002, com a organização apoiada por seis Centros Colaboradores de Alimentação e Nutrição (CECAN) e pelas Áreas Técnicas de Alimentação e Nutrição (ATAN) nos estados e no Distrito Federal. Foram formados 280 técnicos em todo o país (coordenadores e profissionais
1 Ministério da Saúde, Brasília, Brasil.

Correspondência M. F. C. C. Carvalho Ministério da Saúde. Av. Parque Águas Claras 2675, Brasília, DF 71906-500, Brasil. mfatima.carvalho61@gmail.com 
dos programas Saúde da Família e de Agentes Comunitários de Saúde e das ATAN), em temas como direito humano à alimentação, segurança alimentar e nutricional (SAN) e promoção da alimentação saudável no curso da vida. Como resultado, 22 Unidades Federadas implantaram planos de capacitação em seus municípios, com base nas temáticas propostas pelo Ministério da Saúde, agregadas de temas relevantes para o estado/Distrito Federal. Nesse período, ocorreram também programas de formação de gestores/profissionais de saúde da atenção primária à saúde (APS) 1, com foco principal em vigilância alimentar e nutricional, com o apoio do CECAN da Escola Nacional de Saúde Pública Sergio Arouca, Fundação Oswaldo Cruz (ENSP/Fiocruz) 3,4,5,6.

No período de 2007-2014, destaca-se a parceria com a Fiocruz-Brasília para a formação de profissionais/gestores da APS. Em princípio, foi realizado um Curso de Especialização em Gestão de Políticas de Alimentação e Nutrição, modalidade presencial, adotando um modelo de abordagem para ampliar a compreensão dos gestores sobre a rede de determinação causal da alimentação e nutrição e, assim, responder apropriadamente pela execução, acompanhamento e avaliação de programas/políticas de alimentação e nutrição. Cerca de 100 gestores estaduais e municipais foram formados entre 2007-2009 4,5. Em sequência, considerando-se a necessidade de ampliar a capacidade de formação de gestores, somada à prioridade de qualificar profissionais atuando na APS, foram iniciadas duas especializações no âmbito do programa de Ensino à Distância (EAD) da ENSP/Fiocruz: a Especialização em Gestão de Políticas de Alimentação e Nutrição, cujo objetivo foi qualificar a gestão da PNAN, priorizando profissionais atuando com alimentação e nutrição no Ministério da Saúde e nas SES e Secretarias Municipais de Saúde (SMS). Já a Especialização em Alimentação e Nutrição na Atenção Básica priorizou nutricionistas atuantes nos Núcleos Ampliados de Saúde da Família (NASF) e nas equipes (tradicionais) da APS. Em conjunto, esses cursos formaram 294 especialistas 3,4,5,6.

Outra estratégia, financiada pelo Ministério da Saúde, foi a criação da Rede de Alimentação e Nutrição do Sistema Único de Saúde (RedeNutri), em 2009, um espaço virtual, permanente e colaborativo, para a reflexão e a troca de informações e experiências sobre as estratégias e ações para a implementação da PNAN. Entre seus objetivos estão a contribuição para a expansão e a qualificação das ações de alimentação e nutrição nos diferentes pontos de atenção à saúde e para a educação continuada de profissionais envolvidos na realização destas ações, por meio da oferta de cursos EAD autoinstrucionais. Seu gerenciamento era feito por um Comitê Gestor - Observatório de Políticas de Segurança Alimentar e Nutrição, Universidade de Brasília (OPSAN/UnB); CGAN e a Organização Pan-Americana da Saúde/Organização Mundial da Saúde (OPAS/OMS) no Brasil, a produção do conteúdo cabia ao OPSAN, e sua validação, à CGAN 3,4,5,6.

$\mathrm{Na}$ linha do tempo, essas estratégias de formação se somam, se adequam e são ampliadas na medida da necessidade de investir na qualificação da gestão e na implantação de novas ações, estratégias e temáticas (por exemplo: Estratégia Amamenta e Alimenta Brasil, NutriSUS, Promoção da Alimentação Adequada e Saudável), com ênfase na APS. Houve investimentos substanciais de recursos técnicos e financeiros do Ministério da Saúde em produção de materiais educativos (cadernos temáticos, protocolos guias, instrutivos, manuais) e cursos diversos para profissionais/gestores. Contudo, o alcance de tais iniciativas sempre esteve aquém do que é necessário 3,4,5,6.

No período 2015-2019, foram ampliadas as parcerias com Instituições de Educação Superior (IES), de natureza pública, financiadas por Cartas-Acordo ou Termos de Execução Descentralizada. Em 2018, a CGAN, para alavancar o processo de formação, inovou ao lançar Chamadas Públicas, em parceria com o Conselho Nacional de Desenvolvimento Científico e Tecnológico (CNPq), direcionadas a essas IES, visando a selecionar projetos de formação/pesquisa/extensão, para a prevenção e o controle da obesidade na APS 6 . Ainda que não haja resultados dos projetos habilitados nessas Chamadas, pois estão em andamento, a tendência é de permanência dessa modalidade entre as estratégias para avançar na formação de gestores/profissionais da alimentação e nutrição no SUS, com ênfase na APS 5,6.

A formação deve envolver temas essenciais para a prática cotidiana no SUS - da atenção nutricional, envolvendo trabalhadores que atuam no cuidado em saúde, à gestão, tratando da formulação, planejamento, monitoramento e avaliação de programas e ações de alimentação e nutrição. Ocorre que a dinâmica das relações sociais e econômicas impacta os condicionantes/determinantes de saúde e de SAN e, consequentemente, o perfil de alimentação e nutrição da população. Isso repercute sobre o rol de ações a serem ofertadas pelo SUS e sobre a organização dos processos de trabalho, e novos 
conhecimentos e práticas são requeridos aos gestores/profissionais. Portanto, o processo de formação deve ser contínuo e sistemático - pressuposto básico para a ampliação da capacidade resolutiva de atenção nutricional, em especial na APS.

Isso também é desejável em razão da precariedade de vínculos dos profissionais com o SUS. O vácuo da não implantação de um Plano de Carreira, Cargos e Salários (PCCS) do SUS para servidores das três esferas de governo, tema recorrente nas Conferências Nacionais de Saúde, requer círculos contínuos e sistemáticos de formação.

Para estabelecer programas efetivos de educação permanente é essencial a produção de evidências, não somente sobre o cuidado/assistência nutricional, mas também sobre os aspectos relacionados à gestão pública, ao processo e à organização do trabalho nos diferentes níveis de atenção à saúde. $\mathrm{O}$ que e como fazer? Como organizar e avaliar? Quem envolver? Como formar em escala e de forma qualificada? Com quais ferramentas? São perguntas que permanecem no horizonte da gestão nacional, especialmente considerando-se as discrepâncias e desigualdades que se manifestam nas (in) capacidades administrativas, técnicas, políticas e orçamentárias entres estados, regiões e municípios.

Gerir políticas públicas e programas é quase sempre gerir a escassez, histórica e recorrente, de recursos financeiros, físicos e técnicos. A formação técnica e acadêmica deve estar articulada à realidade complexa do SUS, seus regramentos, problemas e potencialidades, processos e procedimentos e organização. Aliar os saberes e competências técnicas dos nutricionistas, por exemplo, às demandas, ao modo de operar e gerir o SUS (e a PNAN) segue sendo um desafio.

Por essas razões, no que diz respeito à qualificação dos trabalhadores de saúde, a gestão da PNAN segue sendo desafiadora. Aqui se destacam a potencialidade e o diferencial de uma possível "reinvenção" dos CECANs. Iniciativa adotada nos anos 2000, e que vigorou até 2011, impulsionou muitas ações da PNAN, incluindo o desenvolvimento de pesquisas e estratégias de formação e capacitação e apoio à gestão e implementação de programas e ações nas esferas subnacionais do SUS. Localizados em IES públicas, eram parceiros estratégicos, conjugando o tripé ensino/pesquisa/extensão, para desenvolver ferramentas, tecnologias, modalidades e propostas/estratégias inovadoras para articular as necessidades do SUS à formação dos profissionais/gestores em alimentação e nutrição.

Sejam quais forem as estratégias de formação, nenhuma pode prescindir da tecnologia informacional. É preciso dar escala às formações permanentes (em serviço) de equipes multiprofissionais e gestores da APS. O investimento em EAD e plataformas, como a Universidade Aberta do Sistema Único de Saúde (UNA-SUS), usadas mais recentemente, são bastante promissoras. O tamanho do SUS justifica essa afirmação, considerando-se o número de equipes e de profissionais de saúde a serem alcançados.

Para além desses desafios, há de se adicionar outros elementos de reflexão nos processos de construção de planos/programas pedagógicos de formação de gestores/profissionais de saúde que dialoguem com as especificidades, diversidades e iniquidades encontradas nos territórios onde atuam, buscando reduzir os fossos das desigualdades locorregionais, econômicas, sociais, culturais, de gênero, étnico-raciais e geracionais.

A superação desses desafios requer articulação permanente da gestão pública com os centros formadores. Os CECAN podem e devem ser potencializados para gerar evidências e apoiar propostas e estratégias efetivas de qualificação de profissionais/gestores na agenda de alimentação e nutrição no SUS. Para além disso, podem ser instâncias colaborativas, de caráter mais permanente, para apoiar as referências institucionais (estaduais/municipais) de alimentação e nutrição, e impulsionar a gestão local e a qualificação de gestores/profissionais de saúde para a gestão intrassetorial e, primordialmente, intersetorial, a fim de avançarmos - concreta e resolutivamente - na implantação das diretrizes da PNAN, em seu conjunto, devidamente alinhadas às especificidades das políticas, programas e realidades locais. 


\section{Informação adicional}

ORCID: Maria de Fátima Cruz Correia de Carvalho (0000-0002-5412-5314).
1. Departamento de Atenção Básica, Secretaria de Atenção à Saúde, Ministério da Saúde. Política Nacional de Alimentação e Nutrição. 2a Ed. Brasília: Ministério da Saúde; 2007.

2. Departamento de Atenção Básica, Secretaria de Atenção à Saúde, Ministério da Saúde. Política Nacional de Alimentação e Nutrição. 1a Reimpr. Brasília: Ministério da Saúde; 2013.

3. Coordenação-Geral de Alimentação e Nutrição. Ações da Política Nacional de Alimentação e Nutrição. Relatório de gestão da Coordenação-Geral da Política de Alimentação e Nutrição: 2003-2006. Brasília: Ministério da Saúde; 2006.

4. Coordenação-Geral de Alimentação e Nutrição. Relatório de gestão 2007-2010: Coordenação-Geral de Alimentação e Nutrição. Brasília: Ministério da Saúde; 2010.

5. Coordenação-Geral de Alimentação e Nutrição. Relatório de gestão 2011-2014: Coordenação-Geral de Alimentação e Nutrição. Brasília: Ministério da Saúde; 2018.

6. Coordenação-Geral de Alimentação e Nutrição. Relatório de gestão 2015-2018: Coordenação-Geral de Alimentação e Nutrição. Brasília: Ministério da Saúde; 2018.

Recebido em 12/Fev/2021

Versão final reapresentada em 11/Jun/2021

Aprovado em 14/Jun/2021 